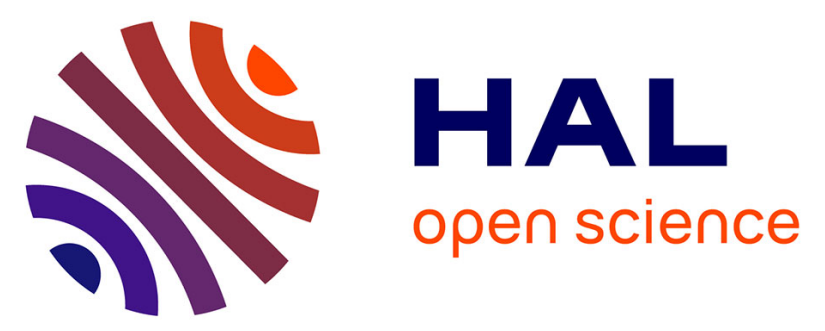

\title{
Interplay between local structure and magnetic properties of graded exchange-coupled Co@FePt nanocomposite films
}

Charles Paleo, Véronique Dupuis, Fabrice Wilhelm, Andrei Rogalev, Olivier Proux, Olivier Boisron, Isabelle Kieffer, Thierry Epicier, Matthieu Bugnet, Damien Le Roy

\section{To cite this version:}

Charles Paleo, Véronique Dupuis, Fabrice Wilhelm, Andrei Rogalev, Olivier Proux, et al.. Interplay between local structure and magnetic properties of graded exchange-coupled Co@FePt nanocomposite films. Physical Review B: Condensed Matter and Materials Physics (1998-2015), 2020, 102, pp.224409. 10.1103/PhysRevB.102.224409 . hal-02975456v2

\section{HAL Id: hal-02975456 \\ https://hal.science/hal-02975456v2}

Submitted on 1 Dec 2020

HAL is a multi-disciplinary open access archive for the deposit and dissemination of scientific research documents, whether they are published or not. The documents may come from teaching and research institutions in France or abroad, or from public or private research centers.
L'archive ouverte pluridisciplinaire HAL, est destinée au dépôt et à la diffusion de documents scientifiques de niveau recherche, publiés ou non, émanant des établissements d'enseignement et de recherche français ou étrangers, des laboratoires publics ou privés. 


\title{
Interplay between local structure and magnetic properties of graded exchange-coupled Co@FePt nanocomposite films
}

\author{
Charles Paleo, ${ }^{1,}$ * Véronique Dupuis, ${ }^{1}$ Fabrice Wilhelm, ${ }^{2}$ Andrei Rogalev, ${ }^{2}$ Olivier Proux,${ }^{3}$ \\ Olivier Boisron, ${ }^{1}$ Isabelle Kieffer, ${ }^{3}$ Thierry Epicier, ${ }^{4}$ Matthieu Bugnet, ${ }^{4}$ and Damien Le Roy ${ }^{1}$ \\ ${ }^{1}$ Institut Lumière Matière, Université Claude Bernard Lyon 1, CNRS, F-69622, Villeurbanne, France \\ ${ }^{2}$ The European Synchrotron Radiation Facility (ESRF), 38000 Grenoble, France \\ ${ }^{3}$ Observatoire des Sciences de l'Univers de Grenoble, \\ Université Grenoble Alpes, CNRS, 38041 Grenoble, France \\ ${ }^{4}$ INSA-Lyon, Université Claude Bernard Lyon 1, CNRS, F-69621 Villeurbanne, France
}

(Dated: November 24, 2020)

\begin{abstract}
Hard-magnetic nanocomposites are attractive materials for integration in various microsystems and for building next generation permanent magnets. However, exploiting their full potential requires to control their microstructure at the nanometre scale. Studying these materials in model systems synthesised by nanofabrication routes gives interesting insights about the interplay between the microstructure and the magnetic performances. Here, by using a combination of mass-selected low energy cluster beam deposition and electron-beam evaporation, we have prepared nanocomposite films where Co nanoinclusions are integrated in a hard magnetic FePt matrix. Local atomic structures and element selective magnetic properties of such nanocomposites have been thoroughly investigated using polarisation dependent hard X-ray absorption spectroscopies. These results demonstrate that magnetically soft inclusions are stabilised at room temperature, emphasising the role of the interdiffusion in the preparation of nanocomposites.
\end{abstract}

\section{INTRODUCTION}

Hard magnetic materials with high magnetisation are attracting a lot of attention for various applications, from spintronics [1] to magnetic recording media [2, 3] or in bulk permanent magnet for energy conversion technologies [4]. The figure of merit of a permanent magnet is the energy product $(\mathrm{BH})_{\max } \leq \frac{1}{4} \mu_{0} M_{s}^{2}$. The limit is attained for an ideal microstructure and an uniaxial texture, which is nearly achieved in $\mathrm{NdFeB}$ sintered and oriented magnets. With an outstanding combination of a relatively large magnetisation $\mu_{0} M_{s}$ of $1.61 \mathrm{~T}$ and a large magnetocrystalline anisotropy leading

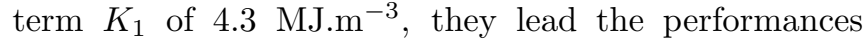
of permanent magnets for about 30 years, approaching the $(\mathrm{BH})_{\max }$ theoretical limit of $516 \mathrm{~kJ} . \mathrm{m}^{-3}$ for these non-nanostructured elements [4]. Designing a hard magnetic material with magnetisation $\mu_{0} M_{s}$ higher than $1.61 \mathrm{~T}$ is a necessary -although not sufficient- condition to surpass the performances of nowadays best rare earth (RE) magnets. Fe-Co alloys could be among the most attractive candidates for permanent magnets, with $\mu_{0} M_{s} \geq 1.81 \mathrm{~T}$ for pure Co and up to $\mu_{0} M_{s}=2.45 \mathrm{~T}$ for $\mathrm{Fe}_{65} \mathrm{Co}_{35}$. In the latter case, it could push the limit of reachable $(\mathrm{BH})_{\max }$ up to nearly $1.2 \mathrm{MJ} . \mathrm{m}^{-3}$, if a square magnetisation loop with a coercive field in excess of $1.2 \mathrm{~T}$ could be achieved $\left(\mu_{0} H_{c} \geq \frac{\mu_{0} M_{s}}{2}\right)$. However, this relies on our ability to significantly increase the relatively low magnetocrystalline anisotropy $\left(K_{1} \leq 20 \mathrm{~kJ} . \mathrm{m}^{-3}\right)[5]$, so far predicted but not fully achieved experimentally [6], even if promising results have recently been reported [7].

\footnotetext{
* charles.paleo@univ-lyon1.fr
}

In addition to these challenges, the risks associated with the sourcing and the fluctuating cost of RE metals due to geostrategic availability, and the pollution during extraction of the raw elements and recycling of the used materials are driving the need to reduce the dependency to the REs in high performance permanent magnets[8$10]$.

An elegant approach to design a magnet with high value of energy product was proposed by Kneller and Harwig, that consists in fabrication of a nanocomposite (NC) materials combining a hard magnetic phase exchange coupled to a strongly magnetic soft phase [11]. The beneficial effect of this approach was initially demonstrated in 1989 by Coehoorn on $\mathrm{Nd}_{2} \mathrm{Fe}_{14} \mathrm{~B}-\mathrm{Fe}_{3} \mathrm{~B}$ ribbons [12]. This finding stimulated further investigations, including theoretical and modelling studies to optimise the NC structure $[11,13]$. In order to develop new magnets with unprecedented performances, the volume fraction of the hard magnetic phase should be limited to the minimum needed to fulfil the coercive field criterion [13] $\mu_{0} M_{s} / 2$ in an ideal uniaxial anistropic case, further extended to realistic cases by Skomski et al.[14]. Under the assumptions of zero magnetic anisotropy in the softer phase and sharp interface between soft and hard magnetic phases, the maximum size of the soft phase regions is twice the size of the domain wall width of the hard phase. Therefore, to exploit the full potential of nanocomposite magnets, one needs to control their microstructure at the nanometre scale, including the size of the soft inclusions, their concentration and the roughness of the interface between the hard and soft phases. In this context, nanocomposite films made from nanofabrication routes are foreseen to bring interesting insights in view of guiding the synthesis of bulk 
systems $[14-16]$.

In this work, we investigate the principle of thin-film nanocomposite magnets of $\mathrm{Co}$ nanometre-sized grains embedded in a hard FePt matrix (named Co@FePt). The fine microstructure analysis requires techniques allowing the chemical distinction of $\mathrm{Fe}$ and $\mathrm{Co}$ at the nanometric scale. The chemically ordered $\mathrm{L} 1_{0}-\mathrm{FePt}$ phase presents a

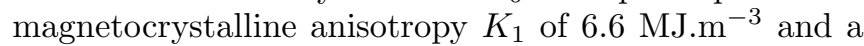
magnetisation of $\mu_{0} M_{s}=1.43 \mathrm{~T}$ (a maximum $(\mathrm{BH})_{\max }$ of $407 \mathrm{~kJ} . \mathrm{m}^{-3}$ ), slightly inferior to the one of $\mathrm{NdFeB}$ [5]. Besides, the use of expensive elements like $\mathrm{Pt}$ for bulk magnets is excluded. However, when it comes to microtechnologies where the fabrication costs generally dominate over the raw material costs, FePt films can be of great interest. Indeed, they offer higher resistance against corrosion, as compared to RE-based films, which makes them more compatible with standard microfabrication processes. NC films of $\mathrm{Co}_{50} @(\mathrm{FePt})_{50}$ would potentially exhibit $28 \%$ higher $(\mathrm{BH})_{\max }$ than the $\mathrm{L} 1_{0}-\mathrm{FePt}$ alone.

\section{METHODS}

\section{A. Sample Preparation}

These Co@FePt transition metal-based nanocomposite are made from Co soft clusters embedded in a $\mathrm{L} 1_{0}-\mathrm{FePt}$ matrix: the clusters are pre-formed in the gas phase using a laser vaporisation source working in a Low Energy Cluster Beam Deposition (LECBD) system[17]. Deposited in a soft landing regime, the clusters are not fragmented or distorted at the impact on the substrate. Moreover, a quadrupolar electrostatic mass-deviator allows to tighten the size dispersion and to obtain a Gaussian distribution centred at a desired mean value ranging from 2 to $10 \mathrm{~nm}$ [18].

The ultra-high vacuum chamber (base pressure in the range of $10^{-10} \mathrm{mbar}$ ) is equipped with an electron-beam evaporator with three targets ( $\mathrm{Fe}, \mathrm{Pt}$ and $\mathrm{Co}$ ) that serve to grow films (for matrix, references and nanofilms samples) by successive atomic depositions.

To prepare the nanocomposites samples, a first layer of Co clusters is deposited on a Si substrate, then a $1.15 \mathrm{~nm}$ film of Fe, then a $1.40 \mathrm{~nm}$ third film of Pt from electrongun evaporation. These three steps are repeated six times in total (see Supporting Information [19] Fig. S1a). CoFePt nanofilms (NF) samples are prepared entirely by electron gun evaporation, with the same atomic Co content as NC samples (Fig. S1b). Finally, a L1 $1_{0}-\mathrm{FePt} \mathrm{NF}$ without Co was prepared as a reference ( $\mathrm{L} 1_{0}$-FePt ref.).

The total thickness of the samples is between $15.3 \mathrm{~nm}$ (for $\mathrm{L} 1_{0}$-FePt ref.) and $21.3 \mathrm{~nm}$ (for $30 \%$ Co samples in atomic proportion).

The formation of the magnetically hard $\mathrm{L} 1_{0}$ phase is thermally activated upon high vacuum annealing at $700^{\circ} \mathrm{C}$ for $20 \mathrm{~min}[20-22]$ (Fig. S1c). The chemical order- ing has been observed for annealing temperatures higher than $650^{\circ} \mathrm{C}$ with the appearance of the (001) line owing to stacking of pure element planes. The $(001) /(110)$ peak intensity ratio is greater than the one expected for an isotropic polycrystalline system; indicating a partial $c$-axis texture. However, the co-existence of strong (111) and (001) peaks indicate a relatively large angular dispersion in the preferential orientation. Note that similar observations were reported previously in films prepared from alternative deposition of $\mathrm{Fe}$ and $\mathrm{Pt}$ thin layers[20] where the authors assigned the preferential $c$-axis texture to the growth process.

\section{B. TEM Analysis}

Bright field transmission electron microscopy (TEM) imaging of the mass-selected Co clusters protected by a carbon film was performed in a Jeol 2100 HT, operated at $200 \mathrm{kV}$. The TEM-energy-dispersive X-ray (TEM-EDX) experiments were performed in a FEI Titan ETEM G2 60-300, operated at $300 \mathrm{kV}$. For this purpose, a FePt/Co clusters/FePt specimen was deposited by LECBD directly on the $\mathrm{Si}_{3} \mathrm{~N}_{4}$ membrane of a DENSsolutions Wildfire MEMS chip, maintained at room temperature in the present study.

\section{XMCD, XLD and XANES analyses}

Polarisation dependent X-ray absorption, X-ray linear dichroism (XLD) and X-ray magnetic circular dichroism (XMCD) experiments have been performed at the ID12 beamline [23] of the European Synchrotron Radiation Facility (ESRF). The X-ray source for these measurements was the first harmonic of APPLE-II type helical undulator (HU-38) in a pure helical mode. The X-ray beam was monochromatised with a fixed-exit double-crystal monochromator equipped with a pair of $\mathrm{Si}(111)$ crystals. The X-ray absorption spectra were recorded using the total fluorescence yield detection mode. The Xray fluorescence signal from the samples were collected in the back-scattering geometry, using Si photodiodes. The XLD signal is obtained as a difference between two absorption spectra measured with X-ray polarisation vector being either parallel or nearly perpendicular (80 degrees) to the sample surface. To minimise any eventual experimental artefacts, we kept the sample orientation constant and polarisation of X-rays using a $0.9 \mathrm{~mm}$ thick diamond quarter wave plate. X-ray quarter wave plate allowed us to transform the incoming circular polarised beam into two orthogonal linearly polarised beams at each energy point of the scan. The absorption was recorded from the same part of the sample for both polarisations. The local magnetic properties of the $\mathrm{Fe}$ and $\mathrm{Co}$ sites were studied using XMCD method at the Fe and Co K-edge. Measurements were performed at $295 \mathrm{~K}$ and under an applied magnetic field of $4 \mathrm{~T}$ provided by a supercon- 
ducting solenoid. The angle between the sample surface and the applied field is 10 degrees. The XMCD spectra were obtained as the difference between two consecutive X-ray absorption near edge structure (XANES) spectra recorded with opposite photon helicities of the incoming photons. Measurements were performed for both directions of applied magnetic field parallel and anti-parallel to the incoming X-ray wave vector in order to ensure the absence of experimental artefacts. The element-selective magnetisation curves were recorded by monitoring the intensity of XMCD signal as a function of an applied magnetic field.

\section{EXAFS Fitting}

The software suite Demeter[24], which includes Athena (data normalisation) and Artemis (simulation), was used. This software allows the fitting of the extended X-ray absorption fine structure (EXAFS) formula signal, allowing for each atomic shell $j$ around the target atom $i$ the determination of $N_{j}$ the coordination number of the atom $i, S_{i}^{2}$ the reduction factor from multi-electronic effects, $F_{j}$ the backscattering amplitude, $\sigma_{j}^{2}$ the Debye-Waller factor, $k=\sqrt{\frac{2 e m_{e}}{\hbar^{2}}\left(E-E_{0}\right)}$ the photoelectron wave vector, $\lambda$ the mean free path of the photoelectron, $r_{j}$ the interatomic distance and $\phi_{i j}$ the phase shift.

After the extraction of the EXAFS signal $\chi(k)$ from the absorption signal, its FT $(\chi(R))$ has been restricted to fit the neighbouring of the absorbing atom. The fitting is done by modelling the expected spectrum with FEFF6[25, 26], choosing the main paths of the photoelectron, then fitting with more paths as the fitting window is increased to higher radial distances. The $S_{i}^{2}$ parameter, which accounts for the relaxation of the other electrons of the absorbing atom[27], is kept fixed in the fits to a value obtained using pure reference foils of each element: 0.73 for $\mathrm{Fe}, 0.70$ for $\mathrm{Co}$, and 0.84 for $\mathrm{Pt}$. The fitted parameters are: the energy edge $E_{0}$, allowed to slightly vary according to the sample, the radial distances $R_{e f f}$, to account for distortion of the tested crystal structure $R$ along the $a$ and $c$-axis (while maintaining the cell volume for cubic structures), and the Debye-Waller $\sigma$.

The $\mathrm{L} 1_{0}$-FePt ref. sample provided a calibration mean to the procedure which led to the best fit with the $\mathrm{L} 1_{0}$ structure for this film.

\section{RESULTS}

The mean size of mass-selected Co clusters is around $7.9 \mathrm{~nm}$ in diameter as-deposited, as obtained from TEM observation (Fig. 1a and b). To verify the cluster diameter in the FePt matrix, a single Co cluster layer embedded between two FePt on a $\mathrm{Si}_{3} \mathrm{~N}_{4}$ substrate has been prepared to perform EDX images, provided in Fig. 1c, where the

clusters observed in the metallic matrix have a size compatible with the diameter found by TEM imaging.

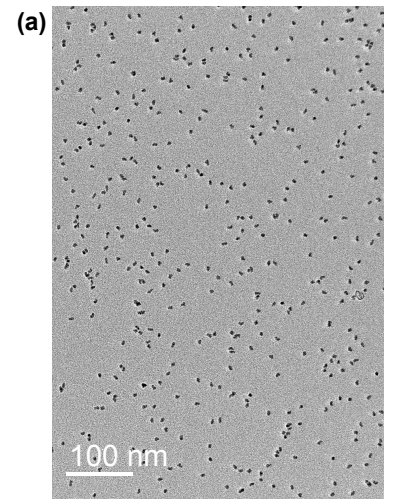

(c)
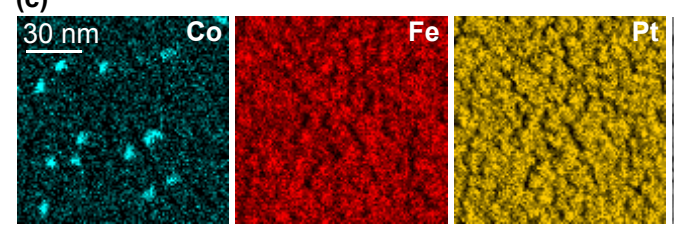

FIG. 1. (a) Bright field TEM image of the mass-selected Co clusters protected by a C film. (b) Size histogram deduced from TEM observations along with the Gaussian fit. (c) EDX images of a FePt/Co clusters/FePt sample on a $\mathrm{Si}_{3} \mathrm{~N}_{4}$ substrate, along with the annular dark-field scanning transmission electron microscopy (STEM-ADF) image. Both measurements give consistent cluster diameter pre-annealing, even when embedded in metallic matrix. The distribution of Fe and Pt is not perfectly homogeneous but reveals "cloudslike" formations, similar to thin films deposition, which most likely disappear when the thickness increases[28].

\section{A. Magnetic properties}

The magnetic properties of Co@FePt nanocomposites were studied by superconducting quantum interference device (SQUID) magnetometry and XMCD experiments performed at room temperature.

Fig. 2 presents the magnetisation curves of $\mathrm{NC}$ and $\mathrm{NF}$ containing $30 \% \mathrm{Co}$, and a reference $\mathrm{L} 1_{0}-\mathrm{FePt}$ film ( $\mathrm{L} 1_{0}-\mathrm{FePt}$ ref). On the one hand, both $\mathrm{NC}$ and $\mathrm{NF}$ samples have higher saturation magnetisation than the $\mathrm{L} 1_{0}$-FePt ref., which can be attributed to the higher magnetisation of Co as compared to FePt. On the other hand, the NC sample exhibits a higher coercivity than the NF, which is magnetically soft as expected for cubic $\mathrm{L}_{2}$ phase. The remanence magnetisation, measured in plane, is relatively high in all cases (superior to $80 \%$ ), and indicates only partial out-of-plane $c$-texture. Despite the higher magnetisation value of the NC, the estimated energy product $(\mathrm{BH})_{\max }$ is lower compared to the one in $\mathrm{L}_{0}$-FePt ref. because of lower coercive field (Table I). 


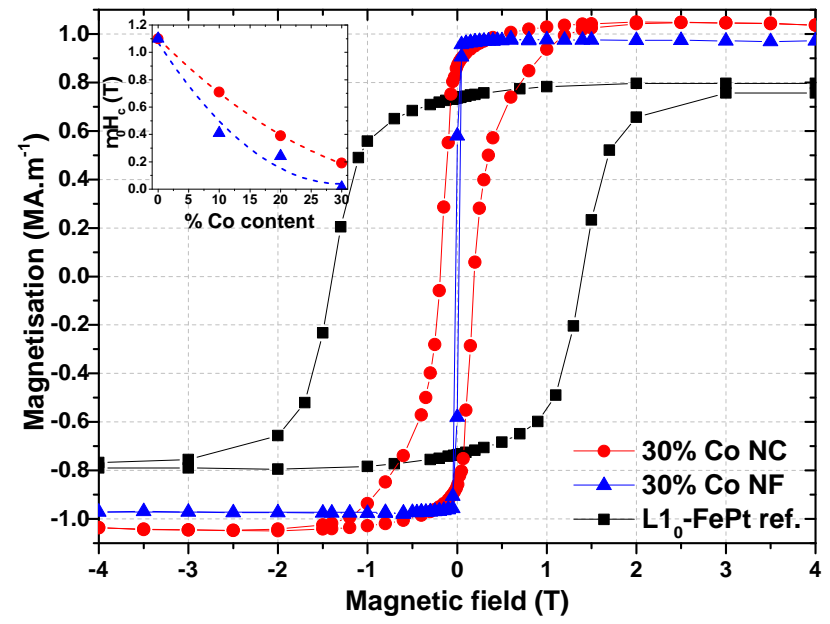

FIG. 2. Hysteresis loops at $300 \mathrm{~K}$ for two samples with the same Co content and the FePt matrix without Co. The NC sample has higher saturation magnetisation than the bare $\mathrm{L} 1_{0}$-FePt ref. which is brought by the Co, but keeps a sizeable coercivity whereas the NF are magnetically soft. The inset shows the dependence of the coercive field $\mu_{0} \mathrm{H}_{c}$ with the Co content for $\mathrm{NF}$ and $\mathrm{NC}$ (the dashed lines are a guide for the eye).

In the ideal case of a sharp interface between the hard FePt and the soft Co phases, element specific studies permit to separately probe both components. The dependence of the XMCD signal as a function of the applied magnetic field (Fig. 3) shows that Fe and Co exhibit the same behaviour and switch at the same field value for every concentration (in the studied range $0-30 \% \mathrm{Co}$ ), indicating a strong coupling. A slight difference is observed in the coercive field values extracted from XMCD (Fig. 3) and SQUID (Fig. 2) measurements, on the same samples. This is attributed to the difference in the probed regions, larger in the case of SQUID measurements.

Isolated face-centred cubic (fcc) Co clusters with a diameter of $7.9 \mathrm{~nm}$ are expected to be superparamagnetic at room temperature and, therefore, magnetically soft [30]. Here, there is no kink at low field on the magnetisation curve, showing that the whole film behaves like a single magnetic phase and there is no indication of the presence of a secondary magnetic phase. However, it does not give access to the degree of interdiffusion at the cluster-matrix interface, which may lead to different

TABLE I. Coercive field and energy product for a NC, a NF, the $\mathrm{L} 1_{0}-\mathrm{FePt}$ ref., and a $\mathrm{Nd}_{2} \mathrm{Fe}_{14} \mathrm{~B}$ random bonded magnet from Coey [29].

\begin{tabular}{cccc}
\hline \hline & $\mu_{0} H_{c}(\mathrm{~T})$ & $M_{s}\left(\mathrm{MA} . \mathrm{m}^{-1}\right)$ & $(\mathrm{BH})_{\max }\left(\mathrm{kJ}^{-3}\right)$ \\
\hline $30 \%$ Co NC & 0.18 & 1.0 & 63 \\
$30 \%$ Co NF & 0.01 & 1.0 & $<1$ \\
L1 $1_{0}-\mathrm{FePt} \mathrm{ref.}$ & 1.4 & 0.8 & 119 \\
$\mathrm{Nd}_{2} \mathrm{Fe}_{14} \mathrm{~B}$ & $\sim 0.8$ & 1.3 & 63 \\
\hline \hline
\end{tabular}

intermixing states (as illustrated in Fig. S2). Such an interdiffusion is expected to result in a modulation of the composition with an intermediate diffused layer between a Co core and a FePt matrix that could be denoted as $\left(\mathrm{Co} @ \mathrm{Co}_{x} \mathrm{Fe}_{y} \mathrm{Pt}_{z} @ \mathrm{FePt}\right)$. The ultimate stage of interdiffusion would be a homogeneous alloyed film, which is rather unlikely as the magnetic properties of $\mathrm{NC}$ differ significantly from the reference homogeneous NF.

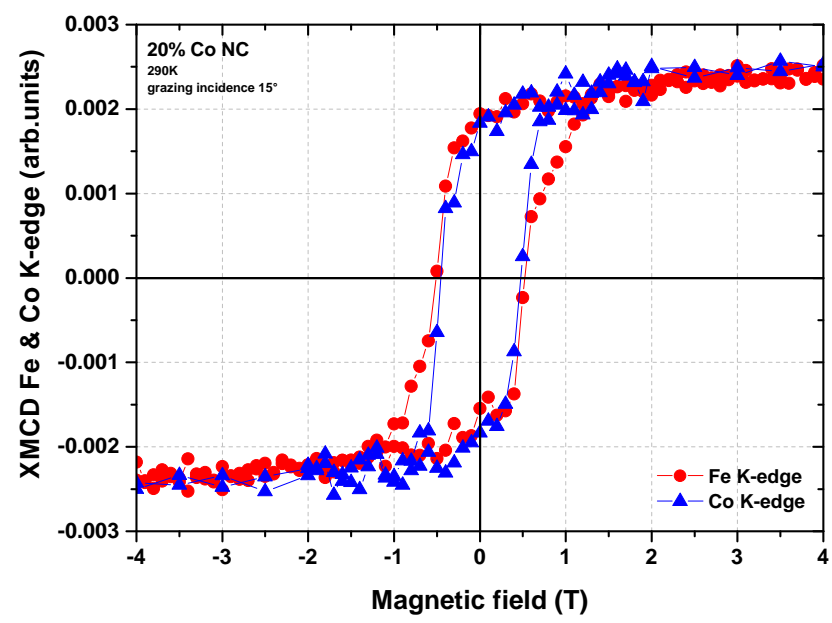

FIG. 3. Hysteresis loops at $290 \mathrm{~K}$ of a $20 \%$ Co NC sample at $\mathrm{Fe}$ and $\mathrm{Co} \mathrm{K}$-edges. Both elements show the same coercivity. Note that the deviation of Fe and Co magnetic moment curves between 0.5 and $1 \mathrm{~T}$ is attributed to lower density of Co clusters at the edge of the probed area in grazing incidence.

Apart from the latter hypothesis of a homogeneous configuration, definitely ruled out by SQUID magnetometry measurements, it would be very difficult to discern between non cluster-matrix interdiffusion and $\mathrm{Co} @ \mathrm{Co}_{x} \mathrm{Fe}_{y} \mathrm{Pt}_{z} @ \mathrm{FePt}$ by TEM imaging. X-ray absorption spectroscopy (XAS) studies have thus been performed to clarify the local structural properties of the samples.

\section{B. Structural properties}

XANES and XMCD signals at the Co K-edge are presented in Fig. 4, for a 20\% Co NC sample and references Co hexagonal close-packed (hcp) and $\mathrm{L} 1_{0}$-CoPt. The $20 \%$ Co NC XANES signal is very different from a Co hcp signal, which shows an important dip on $\mathrm{A}$ and a $\mathrm{B}_{2}$ peak at higher energy [31], whereas the $\mathrm{NC}$ sample and references have a symmetric $\mathrm{B}_{1}$ and $\mathrm{B}_{2}$ feature close to one another, characteristic of face-centred tetragonal (as in $\mathrm{L} 1_{0}-\mathrm{FePt}$ and $\mathrm{L} 1_{0}-\mathrm{CoPt}$ ) alloyed phases[31, 32]. This splitting suggests some alloying of $\mathrm{Co}$ with $\mathrm{Fe}$ and $\mathrm{Pt}$ from the matrix in the $\mathrm{NC}$ sample, as expected for the $\mathrm{L}_{1} 0^{-} \mathrm{CoPt}$ reference. Nevertheless, the $\mathrm{B}_{1}-\mathrm{B}_{2}$ splitting is also present in Co hcp [33, 34], and EXAFS measurements were performed to better understand the structure of the NC sample, and will be detailed later. The XMCD signal 
of the $\mathrm{NC}$ is close to the $\mathrm{L} 1_{0}$-CoPt signal, demonstrating that Co is not in pure metallic state, neither hcp nor fcc.

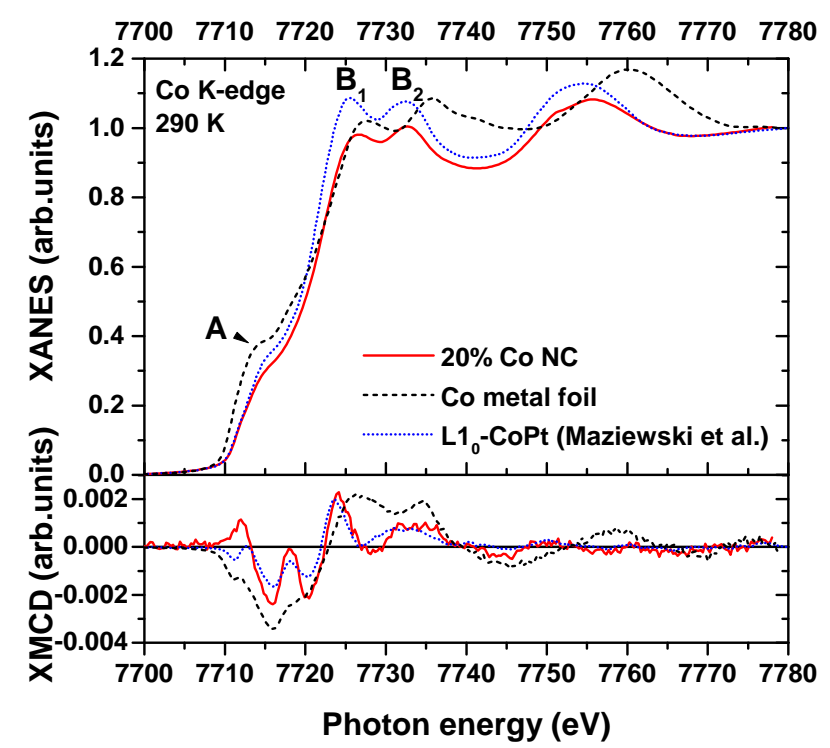

FIG. 4. XANES and XMCD signal at the Co K-edge of a $20 \%$ Co NC, a Co hcp metallic foil (from private communication with $\mathrm{A}$. Rogalev and $\mathrm{F}$. Wilhelm) and a $\mathrm{L} 1_{0}$-CoPt reference (from Maziewski et al. [35]).

XLD measurements at the Fe K-edge for $20 \%$ Co $\mathrm{NC}, \mathrm{NF}$ and the $\mathrm{L} 1_{0}-\mathrm{FePt}$ reference (Fig. S3) reveal significant XLD signal which proves a -at least partialtexturation of the sample, favoured by the alternative stacking of the layers[20]. The XANES peaks $\mathrm{B}_{1}$ and $\mathrm{B}_{2}$ appears asymmetric only for NF sample $\left(\mathrm{B}_{2}\right.$ feature higher than $\mathrm{B}_{1}$ ), which is consistent with an enrichment in Co for NF samples, as observed in other studies[36] and the XANES of the hcp Co.

The shoulder A, mainly due to Co and Fe $4 p$ orbitals[32] is larger for the NF, as expected for a more complete mixing in the NF as compared to in the NC.

To study the local structure of the samples, XAS measurement have been performed to obtain the specific EXAFS signature at the $\mathrm{Fe}$ and $\mathrm{Co} \mathrm{K}$-edges and $\mathrm{Pt} \mathrm{L}_{3}$-edge (see SI methods for details [24, 27]). Fig. 5 presents the modulus and real part of the Fourier transform (FT) of the EXAFS signal for 30\% Co NC and NF, giving an additional qualitative indication of different local environments in both systems. The comparison to the $\mathrm{L} 1_{0}-\mathrm{FePt}$ ref. (Fig. S4), shows that the NC is much closer to this phase at the Fe K-edge. This is an argument in favour of a stronger Co-matrix diffusion in the NF than in the $\mathrm{NC}$, which could also explain the low coercivity obtained on this NF sample. As Co and Fe show similar binary phase diagram with $\mathrm{Pt}[37]$, the more atomic interdiffusion occurs, the more the stable phase shifts towards the higher $\mathrm{Co}+\mathrm{Fe}$ content values, leading to a magnetically soft $\mathrm{L}_{2}-\mathrm{X}_{3} \mathrm{Pt}$ (with $X=\mathrm{Co}, \mathrm{Fe}$ ) when Co content is high enough (as in 30\% Co samples).
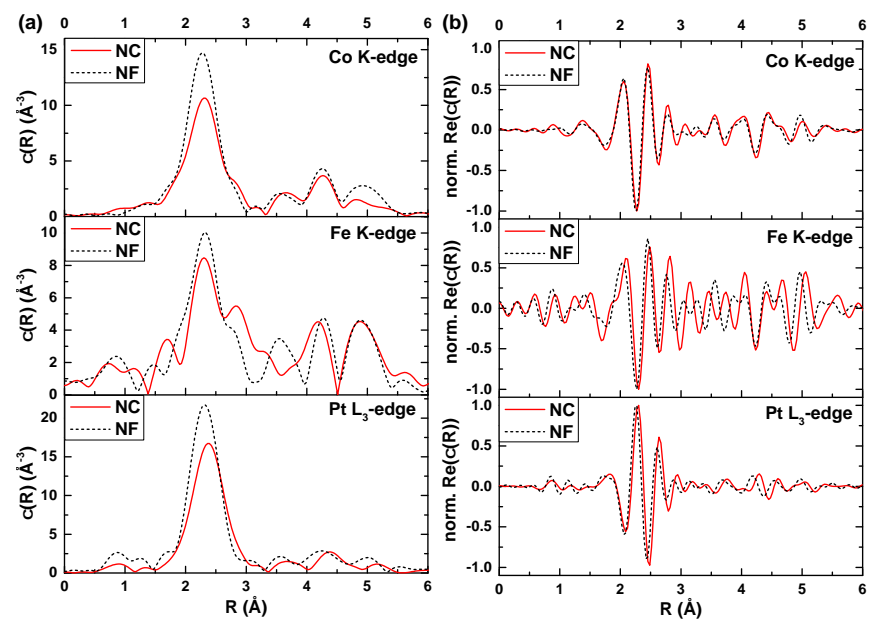

FIG. 5. Comparison of the magnitude (a) and real part of the FT (b) of the EXAFS signal for 30\% Co NC and 30\% Co $\mathrm{NF}$ at each edge.

Two different kinds of simulation have been realised to analyse the spectra: a first fit is performed on a wide radial distance (typically between 1.5 and $5.5 \AA$ ) at each edge, with different possible crystal alloy phases, by setting the nature and number of neighbouring atoms $N_{j}$. The best fits from this method for each edge is provided on Table II (detailed EXAFS results are provided in the SI Tables).

For the $30 \%$ NC sample, the EXAFS simulations at the Fe K-edge and $\mathrm{Pt} \mathrm{L}_{3}$-edge lead to $\mathrm{L}_{0}$-FePt structure. On the contrary, for the NF sample, the optimised structure is a mixed $\mathrm{X}_{3} \mathrm{Pt}$ structure at the $\mathrm{Fe} \mathrm{K}$-edge and $\mathrm{Pt} \mathrm{L}_{3}$ edge, confirming the strong Co-matrix intermixing in this sample. Distinguishing Fe from Co neighbouring atoms based on EXAFS measurements is a difficult task (as $\Delta Z=1)$. Nevertheless, the fact that the $\mathrm{Co}_{3} \mathrm{Pt}$ phase fits only at Co K-edge in the NC samples (Fig. 6) while $\mathrm{L} 1_{0}$-FePt phase fits at $\mathrm{Fe} \mathrm{K}$ and $\mathrm{Pt} \mathrm{L}_{3}$-edge suggests the persistence of Co-rich regions.

To quantify atomic interdiffusion between cluster and matrix in the NC, another fit is performed at the Co Kedge on NC samples, by choosing small radial distances (between 1.5 and $3.0 \AA$ ) to limit to the first neighbours of the Co atoms. The fit is realised with Co (accounting for both $\mathrm{Co}$ or $\mathrm{Fe}$ atoms) and $\mathrm{Pt}$ atoms, initially at positions found from the previous $\mathrm{Co}_{3} \mathrm{Pt}$ fit. The coordination numbers $N_{j}$ are then set free (but restraining to $N_{C o, F e}+$ $N_{P t}=12$ ) and have been obtained by the fit at different

TABLE II. Structure giving the best fits for a window between 1.5 and $5.5 \AA(X=C o, F e)$.

\begin{tabular}{lcc}
\hline \hline Best fit at & $\mathrm{NC}$ & $\mathrm{NF}$ \\
\hline Co K-edge & $\mathrm{X}_{3} \mathrm{Pt}$ & $\mathrm{X}_{3} \mathrm{Pt}$ \\
Fe K-edge & $\mathrm{L} 1_{0}-\mathrm{FePt}$ & $\mathrm{X}_{3} \mathrm{Pt}$ \\
$\mathrm{Pt} \mathrm{L} \mathrm{L}_{3}$-edge & $\mathrm{L} 1_{0}-\mathrm{FePt}$ & $\mathrm{X}_{3} \mathrm{Pt}$ \\
\hline \hline
\end{tabular}



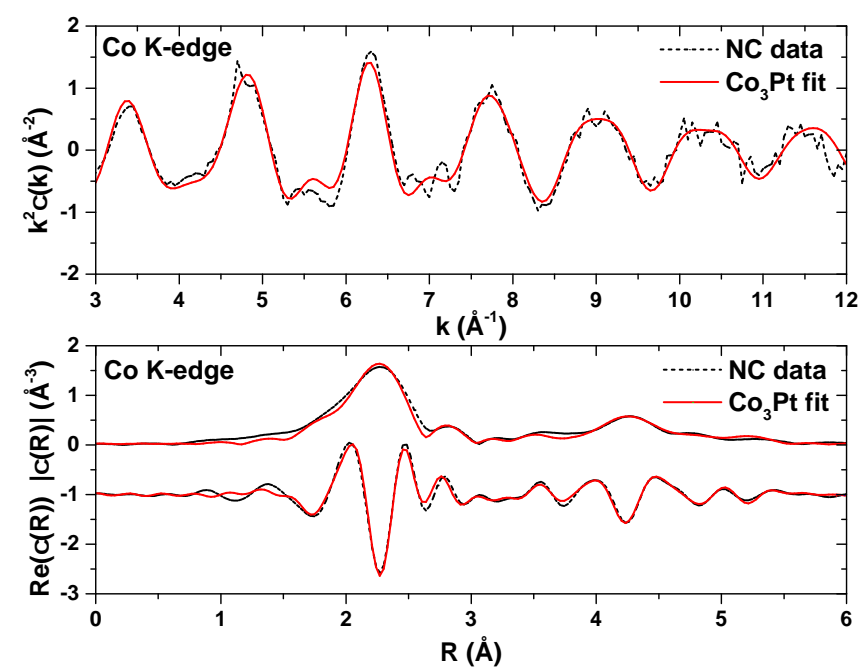

FIG. 6. Weighted EXAFS oscillations, magnitude of the filtered signal, and real part of the FT of the $30 \%$ Co NC sample at the Co K-edge, along with the $\mathrm{Co}_{3} \mathrm{Pt}$ fit.

angles. The number of $\mathrm{Co}-\mathrm{Co}$ bonds is found to be in average centred on $\mathrm{N}_{\mathrm{Co}-\mathrm{Co}}=4.2$ (Fig. S5).

In addition, a diffusion of the cluster is simulated from an initial truncated-octahedron Co cluster (stable shape of fcc clusters[38]) of 11 atoms/ridge (which size corresponds to the size observed by TEM) inserted in a FePt matrix. A simple iterative diffusion algorithm is then applied to every atom by giving a $50 \%$ probability of swapping its place with a neighbouring atom. The mean number of Co-Co bonds is then calculated and compared to the number obtained by EXAFS fitting. These steps are repeated until the coordination number of the model is equal or lower than the one obtained from the EXAFS. It results that this 4.2 coordination number can occur when matrix atoms reach the centre of the cluster (Fig. S6), but the Co concentration remains above $75 \%$ in a $1.8 \mathrm{~nm}$ radius at least.

For NC, the important criterion is the core size of the soft inclusion. According to the Co-Pt (or Fe-Pt) phase diagrams, the edge of the $\mathrm{L} 1_{0}$ phase is at least $35 \%$ content in $\mathrm{Co}$ (or Fe)[37]. Therefore, with Co-content clusters embedded in hard $\mathrm{L}_{0}$ matrix, the soft inclusion of $\mathrm{X}_{3} \mathrm{Pt}$ remains around $7.4 \mathrm{~nm}$ diameter after annealing (Fig. S7).

\section{CONCLUSION}

Magnetic measurements of the composition dependence of the coercive field as well as the EXAFS fitting and simulation treatment prove that, after annealing at $700^{\circ} \mathrm{C}$ for $20 \mathrm{~min}, \mathrm{NC}$ still present high Co content inclusions well coupled to the matrix, contrary to the
NF where the interdiffusion is complete. To explain such result, one can first mention that clusters have a well-defined shape as prepared and are particularly stable[39]. Secondly, for the same amount of Co in a sample, the thickness of the Co layer in NF $(1.0 \mathrm{~nm}$ for $30 \% \mathrm{Co}$ ) is smaller than the radius of a cluster as prepared, favouring the total Co-atomic diffusion in matrix during annealing. This lower diffusion in NC allows the matrix to stay in the $\mathrm{L} 1_{0}$ stability domain, keeping the magnetic hardness of the sample.

Moreover, the cluster diffusion simulation does not take into account the different miscibility as well as the different sizes of $\mathrm{Fe}, \mathrm{Co}$, or Pt atoms, which can lead to different diffusion coefficients[37]. As such, heavy Pt atoms of the matrix are less likely to diffuse into the Co cluster. The crystal distortions of the fcc Co-rich regions in the face-centred tetragonal (fct) $\mathrm{L} 1_{0}$ matrix have also been neglected in the simulation, but are revealed by the EXAFS fitting.

One of the key factor to improve the $(\mathrm{BH})_{\max }$ is a better control of the cluster-matrix interdiffusion: with thinner layers of $\mathrm{Fe}$ and $\mathrm{Pt}$ and a larger number of repetition, the annealing to form the $\mathrm{L} 1_{0}$ phase can be achieved at lower temperature and faster. Moreover the maximum of coercivity for non-epitaxial thin films is not reached for $\mathrm{Fe}_{50} \mathrm{Pt}_{50}$, but rather for $\mathrm{Fe}_{53} \mathrm{Pt}_{47}[22,40]$. Although pure Co clusters provide the opportunity to visualise soft and hard phases individually, they are not in favour of a high $(\mathrm{BH})_{\max }$, as the highest moment per atom is reached for $\mathrm{Fe}_{65} \mathrm{Co}_{35}[5]$. Finally, the texturation of the hard magnetic matrix could be improved using a $\mathrm{MgO}$ crystal substrate. These Co@FePt samples are a proof of concept of NC permanent magnets, stabilising the moments of the soft clusters at room temperature.

\section{ACKNOWLEDGMENTS}

The authors thank Florent Tournus (iLM laboratory) for his help on the cluster modelling, Nicholas Blanchard (iLM laboratory) for TEM imaging, and José Penuelas (STMS/INL) for the XRD measurements. The TEMEDX experiments were performed at the Consortium Lyon St-Etienne de Microscopie. XMCD, XLD, XANES and EXAFS measurements were made at ESRF, on beamline ID12 and French CRG BM30B FAME for the latter. This work is supported by the ANR through the collaborative project SHAMAN (ANR-16-CE09-0019), and the doctoral school ED PHAST 52. The authors are also grateful to the ILMTECH platform for clusters synthesis at PLYRA and SQUID measurements at the Centre de Magnétométrie de Lyon. 
[1] T. N. A. Nguyen, Y. Fang, V. Fallahi, N. Benatmane, S. M. Mohseni, R. K. Dumas, and J. Åkerman, $[\mathrm{co} / \mathrm{pd}]-$ nife exchange springs with tunable magnetization tilt angle, Applied Physics Letters 98, 172502 (2011).

[2] D. Mitin, M. Wachs, N. Safonova, O. Klein, and M. Albrecht, Exchange coupled 110 fecupt/fe heterostructures: Magnetic properties and reversal behavior at elevated temperatures, Thin Solid Films 651, 158-162 (2018).

[3] B. Ma, G. Y. Situ, H. G. Chu, and J. P. Wang, Exchange coupled composite fept/tbco/[co/ni]n films with an tbco interlayer, AIP Advances 7, 056508 (2017).

[4] J. Coey, Perspective and prospects for rare earth permanent magnets, Engineering 6, 119-131 (2020).

[5] J. M. D. Coey, Magnetism and Magnetic Materials, 1st ed. (Cambridge University Press, 2001).

[6] T. Burkert, L. Nordström, O. Eriksson, and O. Heinonen, Giant magnetic anisotropy in tetragonal feco alloys, Physical Review Letters 93, 027203 (2004).

[7] X. Li, L. Lou, W. Song, G. Huang, F. Hou, Q. Zhang, H.-T. Zhang, J. Xiao, B. Wen, and X. Zhang, Novel bimorphological anisotropic bulk nanocomposite materials with high energy products, Advanced Materials 29, 1606430 (2017).

[8] J. Lucas, P. Lucas, T. Le Mercier, A. Rollat, and W. Davenport, Rare earths: science, technology, production and use (Elsevier, 2015).

[9] P. Mcguiness, O. Akdogan, A. Asali, S. Bance, F. Bittner, J. M. D. Coey, N. M. Dempsey, J. Fidler, D. Givord, O. Gutfleisch, and et al., Replacement and original magnet engineering options (romeos): A european seventh framework project to develop advanced permanent magnets without, or with reduced use of, critical raw materials, JOM 67, 1306-1317 (2015).

[10] J. Rial, P. Švec, E. Palmero, J. Camarero, P. Švec, and A. Bollero, Severe tuning of permanent magnet properties in gas-atomized mnal powder by controlled nanostructuring and phase transformation, Acta Materialia 157, 42-52 (2018).

[11] E. Kneller and R. Hawig, The exchange-spring magnet: a new material principle for permanent magnets, IEEE Transactions on Magnetics 27, 3588-3560 (1991).

[12] R. Coehoorn, D. De Mooij, and C. De Waard, Meltspun permanent magnet materials containing fe $3 \mathrm{~b}$ as the main phase, J. Magnet. Magnet. Mater 80, 101-104 (1989).

[13] R. Skomski and J. M. D. Coey, Giant energy product in nanostructured two-phase magnets, Physical Review B 48, 15812-15816 (1993).

[14] R. Skomski, P. Manchanda, P. K. Kumar, B. Balamurugan, A. Kashyap, and D. J. Sellmyer, Predicting the future of permanent-magnet materials, IEEE Transactions on Magnetics 49, 3215-3220 (2013).

[15] X. Rui, J. Shield, Z. Sun, L. Yue, Y. Xu, D. Sellmyer, Z. Liu, and D. Miller, High-energy product exchangespring fept/fe cluster nanocomposite permanent magnets, Journal of Magnetism and Magnetic Materials 305, $76-82$ (2006).

[16] M. Yue, X. Zhang, and J. P. Liu, Fabrication of bulk nanostructured permanent magnets with high energy density: challenges and approaches, Nanoscale 9, 3674-3697 (2017).
[17] V. Dupuis, G. Khadra, A. Hillion, A. Tamion, J. Tuaillon-Combes, L. Bardotti, and F. Tournus, Intrinsic magnetic properties of bimetallic nanoparticles elaborated by cluster beam deposition, Physical Chemistry Chemical Physics 17, 27996-28004 (2015).

[18] R. Alayan, L. Arnaud, A. Bourgey, M. Broyer, E. Cottancin, J. R. Huntzinger, J. Lermé, J. L. Vialle, M. Pellarin, and G. Guiraud, Application of a static quadrupole deviator to the deposition of size-selected cluster ions from a laser vaporization source, Review of Scientific Instruments 75, 2461-2470 (2004).

[19] See supplemental material at [url] for additional figures and tables.

[20] H. Zeng, M. L. Yan, N. Powers, and D. J. Sellmyer, Orientation-controlled nonepitaxial 110 copt and fept films, Applied Physics Letters 80, 2350-2352 (2002).

[21] Y. Liu, T. A. George, R. Skomski, and D. J. Sellmyer, Aligned and exchange-coupled 110 (fe,co)pt-based magnetic films, Journal of Applied Physics 111, 07B537 (2012).

[22] M. H. Hong, K. Hono, and M. Watanabe, Microstructure of fept/pt magneticthin films with high perpendicular coercivity, Journal of Applied Physics 84, 4403-4409 (1998).

[23] A. Rogalev and F. Wilhelm, Magnetic circular dichroism in the hard x-ray range, The Physics of Metals and Metallography 116, 1285-1336 (2015).

[24] B. Ravel and M. Newville, Athena, artemis, hephaestus: data analysis for x-ray absorption spectroscopy using ifeffit, Journal of Synchrotron Radiation 12, 537-541 (2005).

[25] J. J. Rehr and R. C. Albers, Theoretical approaches to xray absorption fine structure, Reviews of Modern Physics 72, 621-654 (2000).

[26] J. J. Rehr, J. J. Kas, M. P. Prange, A. P. Sorini, Y. Takimoto, and F. Vila, Ab initio theory and calculations of x-ray spectra, Comptes Rendus Physique 10, 548-559 (2009).

[27] B. K. Teo, EXAFS: Basic Principles and Data Analysis (Springer Berlin, 2014).

[28] A. I. Figueroa, J. Bartolomé, L. M. García, F. Bartolomé, O. Bunău, J. Stankiewicz, L. Ruiz, J. M. GonzálezCalbet, F. Petroff, C. Deranlot, and et al., Structural and magnetic properties of granular co-pt multilayers with perpendicular magnetic anisotropy, Physical Review B 90, 174421 (2014).

[29] J. Coey, Perspective and prospects for rare earth permanent magnets, Engineering 6, 119-131 (2020).

[30] M. Jamet, W. Wernsdorfer, C. Thirion, D. Mailly, V. Dupuis, P. Mélinon, and A. Pérez, Magnetic anisotropy of a single cobalt nanocluster, Physical Review Letters 86, 4676-4679 (2001).

[31] J.-I. Park, M. G. Kim, Y.-w. Jun, J. S. Lee, W.-r. Lee, and J. Cheon, Characterization of superparamagnetic core-shell nanoparticles and monitoring their anisotropic phase transition to ferromagnetic solid solution nanoalloys, Journal of the American Chemical Society 126, 9072-9078 (2004).

[32] N. M. Souza-Neto, A. Y. Ramos, H. C. N. Tolentino, and Y. Joly, Depth dependent local structures in copt thin films, Journal of Physics: Conference Series 190, 012112 (2009). 
[33] Y. A. Kozinkin, A. A. Novakovich, A. V. Kozinkin, R. V. Vedrinskii, Y. V. Zubavichus, and A. A. Veligzhanin, Mechanisms of formation of near-edge fine structure of k x-ray absorption spectra of metallic cu, ni, co (hcp and fcc phases), and cr, Physics of the Solid State 53, 1-5 (2011).

[34] P. Mazalski, I. Sveklo, Z. Kurant, K. Ollefs, A. Rogalev, F. Wilhelm, J. Fassbender, L. T. Baczewski, A. Wawro, and A. Maziewski, Xas and xmcd studies of magnetic properties modifications of $\mathrm{pt} / \mathrm{co} / \mathrm{au}$ and $\mathrm{pt} / \mathrm{co} / \mathrm{pt}$ trilayers induced by ga+ ions irradiation, Journal of Synchrotron Radiation 22, 753-759 (2015).

[35] A. Maziewski, P. Mazalski, Z. Kurant, M. O. Liedke, J. McCord, J. Fassbender, J. Ferré, A. Mougin, A. Wawro, L. T. Baczewski, and et al., Tailoring of magnetism in $\mathrm{pt} / \mathrm{co} / \mathrm{pt}$ ultrathin films by ion irradiation, Physical Review B 85, 054427 (2012).
[36] E. K. Hlil, R. Baudoing-Savois, B. Moraweck, and A. J. Renouprez, X-ray absorption edges in platinum-based alloys. 2. influence of ordering and of the nature of the second metal, The Journal of Physical Chemistry 100, 3102-3107 (1996).

[37] C. J. Smithells, W. F. Gale, and T. C. Totemeier, Smithells metals reference book, 8th ed. (Elsevier Butterworth-Heinemann, 2004).

[38] R. Van Hardeveld and F. Hartog, The statistics of surface atoms and surface sites on metal crystals, Surface Science 15, 189-230 (1969).

[39] R. Ferrando, Structure and properties of nanoalloys, Frontiers of nanoscience (Elsevier, 2016).

[40] M. Watanabe and M. Homma, Perpendicular magnetization of epitaxial fept(001) thin films with high squareness and high coercive force, Japanese Journal of Applied Physics 35, L1264-L1267 (1996). 